\title{
\begin{tabular}{l|l} 
Mibraries & DSpace@MIT
\end{tabular}
}

\author{
MIT Open Access Articles \\ Dense transmit and receive phased arrays
}

The MIT Faculty has made this article openly available. Please share how this access benefits you. Your story matters.

Citation: Chen-Pang Yeang et al. "Dense Transmit and Receive Phased Arrays." Proceedings of the 2010 IEEE International Symposium on Phased Array Systems and Technology (ARRAY), IEEE, 2010. 934-939. Web. (C2010 IEEE.

As Published: http://dx.doi.org/ 10.1109/ARRAY.2010.5613250

Persistent URL: http://hdl.handle.net/1721.1/73137

Version: Final published version: final published article, as it appeared in a journal, conference proceedings, or other formally published context

Terms of Use: Article is made available in accordance with the publisher's policy and may be subject to US copyright law. Please refer to the publisher's site for terms of use. 


\section{DENSE TRANSMIT AND RECEIVE PHASED ARRAYS}

\author{
Chen-Pang Yeang \\ IHPST, Univ. Toronto, ON, Canada \\ chenpang.yeang@utoronto.ca
}

\begin{abstract}
A dense antenna array architecture is developed to ease the circuit requirements of the radio frequency $(R F)$ front-end in beamforming applications. In the architecture, antennas are spaced more closely than required by the sampling principle to exploit the available degrees of freedom. This array structure is analogous to temporally oversampled data conversion systems, which have reduced quantizer resolution requirements. In [1], we have developed a spatial-domain version of $\Delta \Sigma$ quantization, and have shown that with binary quantization for the in-phase and quadrature components of antenna weights, modest amounts of oversampling can reproduce beamforming patterns of interest to practically useful levels of accuracy. In this paper, we incorporate mutual coupling between antennas and impedance matching in the oversampling scheme and analyze their effects on the performance of this dense-array architecture. These effects do not change the validity of the dense array.
\end{abstract}

\section{INTRODUCTION}

Recent progress in millimeter-wave and microwave integrated circuit (IC) manufacturing technology has created new opportunities for the development of novel, low-cost antenna array architectures. The ability to integrate miniature microstrip antennas has expanded the realm of applications for antenna arrays to various embedded communication and sensing systems. In such systems, the associated specifications on RF components such as phase-shifters, oscillators, and amplifiers, can be challenging to meet in a cost-effective manner. In [1], we have developed a dense antenna array to ease such RF circuit requirements without sacrificing performance, and have demonstrated its effectiveness for transmit and receive beamforming. In this paper, we cast the dense array scheme in a more realistic setting in RF design by incorporating mutual coupling between antennas and impedance matching. We also analyze their effects on the performance of the densearray system.

\author{
Gregory W. Wornell, Lizhong Zheng, James Krieger \\ Dept. EECS, MIT, Cambridge, MA, USA \\ \{gww,lizhong, ja17761\}@mit.edu
}

\section{THE TRADITIONAL ARRAY ARCHITECTURE}

In a traditional uniform linear array, two adjacent antennas elements are separated with distance $d$ and all the $N$ antennas form an aperture of length $L$. The "nominal" spacing between elements is $d=\lambda / 2$. As long as $d \leq \lambda / 2$, the maximum number of available degrees of freedom can be realized and grating lobes in beamforming patterns can be avoided. Thus, the minimum number of antenna elements required is typically $N_{\circ}=1+2 L / \lambda$.

Fig. 1 depicts the associated array architecture. A beam pattern can be formed by choosing antenna element weights $w_{n}=a_{n} e^{j \theta_{n}}$. This can be implemented via $N$ amplitude attenuators and $N$ phase shifters. At the transmitter, the RF signal passes through such a beamformer, whose $N$ outputs are directed to $N$ power amplifiers and then $N$ antennas. At the receiver, the $N$ antenna signals that result from the incoming wave pass through $N$ low-noise amplifiers, are phase-shifted, amplitude-adjusted, and superimposed via the beamformer to form the output signal. The associated transmitter and receiver array radiation patterns are, respectively,

$B^{\mathrm{T}}(\psi)=\left|\sum_{n=1}^{N} w_{n} e^{-j n k d \cos \psi}\right|, B^{\mathrm{R}}(\psi) \equiv\left|\sum_{n=1}^{N} w_{n} e^{j n k d \cos \psi}\right|$,

where $k=2 \pi / \lambda$ is the wave number and $\psi$ is the target angle of radiation.

The traditional array architecture poses challenges to circuit design and device technology. Accuracy of the array radiation patterns in (1) depends on the accuracy of $\left\{\theta_{n}\right\}$ and $\left\{a_{n}\right\}$. This implies that the phase shifters in the beamformers must be implemented with high resolution, and the power amplifiers at the transmitter must have high linearity. Although a variety of approaches have been proposed to improve the precision of these components, most remain expensive and complicated; see, e.g., [2-7].

\section{AN OVERSAMPLING ARRAY ARCHITECTURE}

As an alternative to the traditional architecture, we have proposed in [1] to pack more antennas $N$ in the given aperture than would otherwise be required, i.e., $N>N_{\circ}$, for both 


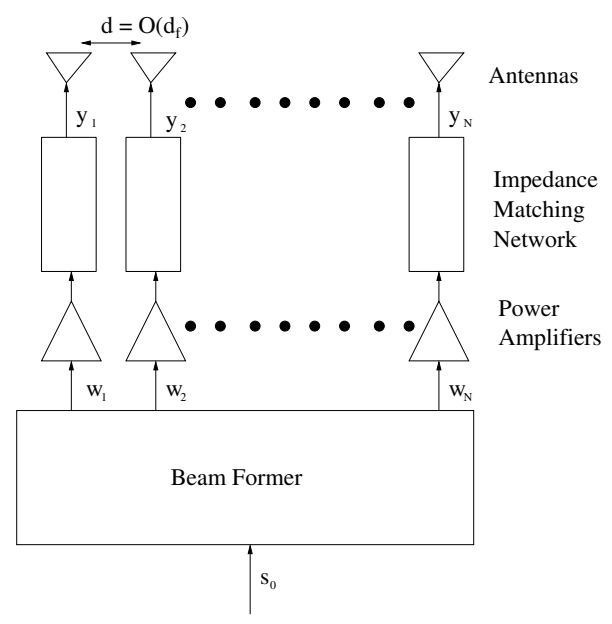

(a) Transmitter

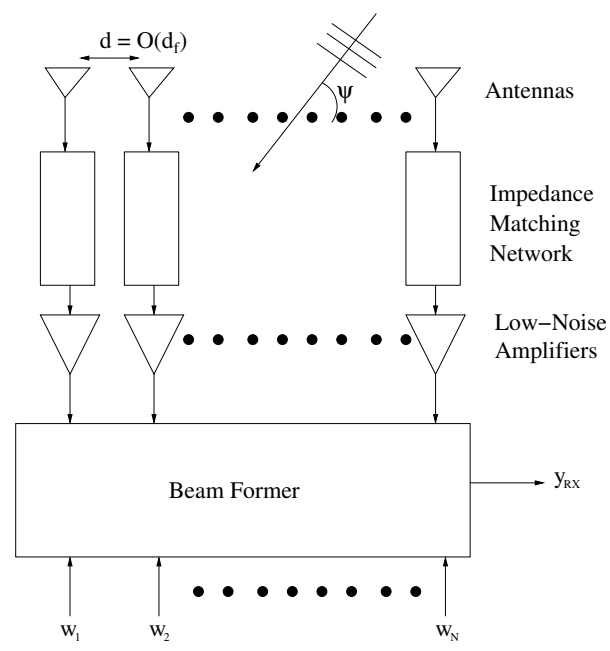

(b) Receiver

Fig. 1. The traditional antenna array architecture.

transmit and receive array configurations. This density is then exploited in a manner analogous to the way temporal oversampling is exploited in data converter design. Just as sampling at a rate exceeding that dictated by the Nyquist criterion reduces quantization resolution requirements, the dense array uses $N$ lower-quality RF components instead of $N_{\circ}$ higher quality ones.

\section{Spatial $\Delta \Sigma$ Quantization}

To reduce the RF component specifications, we constrain our system to use coarsely quantized versions of the weights $w_{n}$, and exploit the oversampling to minimize the effect of such quantization. In particular, we exploit the principle of $\Delta \Sigma$ quantization [8] by directly translating it to the spatial domain. (For other rather different multidimensional extensions of $\Delta \Sigma$ quantization motivated by image processing and space-time coding, see, e.g., [9-11].)

The spatial $\Delta \Sigma$ architecture is depicted in Fig. 2, and involves creating a sequence of quantized weights $v_{n}$ that "act" in a manner asympotically indistinguishable from the desired weights $w_{n}$. Here is the processing procedure: At the $n$th antenna, we obtain the difference between the input of the $(n-1)$ th quantizer and $v_{n-1}$ at the output of the $(n-1)$ th quantizer, corresponding to the $\Delta$ part of the structure. We then add that difference to the desired weight $w_{n}$, corresponding to the $\Sigma$ part of the structure. The result is then quantized using 4-PSQ (phase-shift quantization, where the input is quantized to a constant amplitude and one of four phases: $45^{\circ}, 135^{\circ}, 225^{\circ}, 315^{\circ}$ ), which corresponds to quantizing each of the in-phase and quadrature components to a single bit. As such only crude 4-angle phase shifters and (nonlinear) bilevel power amplifiers are required in the RF front-end.

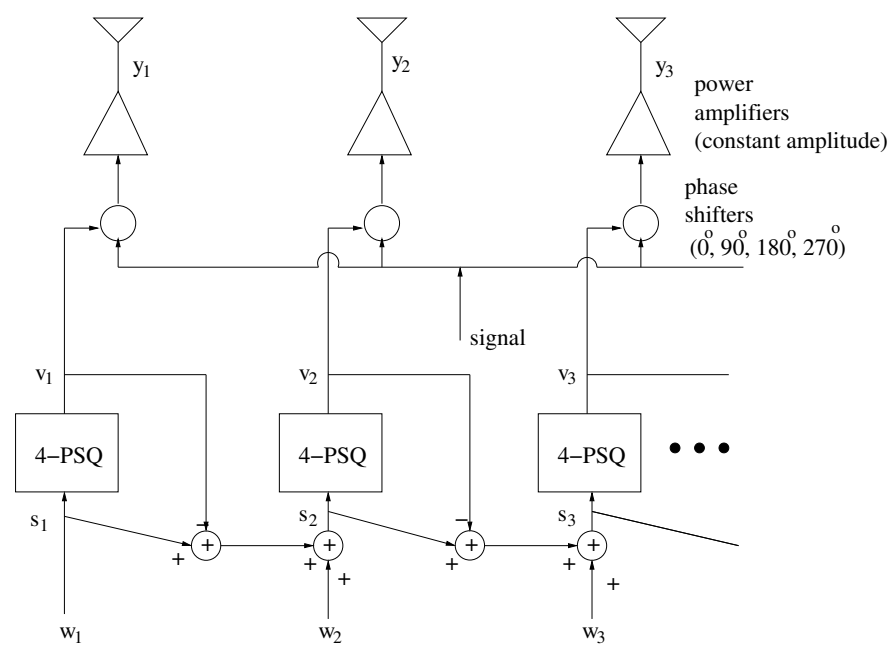

Fig. 2. The spatial $\Delta \Sigma$ architecture at the transmitter. The corresponding receiver architecture is obtained by reversing the beamformer to have multiple inputs and a single output, i.e., the $n$th antenna input from the low-noise amplifier is mixed with $v_{n}$, then all such terms are summed.

\section{Error Analysis for Spatial $\Delta \Sigma$}

Spatial $\Delta \Sigma$ oversampling works in a similar way to traditional temporal $\Delta \Sigma$ oversampling. In the latter, a time series $\left\{w_{n}\right\}$ is quantized into another time series $\left\{v_{n}\right\}$, from which one can recover $\left\{w_{n}\right\}$ by low-pass filtering. That recovery is possible follows from the fact that the feedback structure of $\Delta \Sigma$ forces the average value of the quantized output to track the average input, which has the advantage of suppressing the quantization error spectrum at low frequencies. Similar behavior takes place in the case of our spatial $\Delta \Sigma$ scheme. To see how the associated spatial low-pass filtering arises, note that the transmit and receive array patterns $B^{\mathrm{T}}(\psi)$ and $B^{\mathrm{R}}(\psi)$ in (1) are effectively the Fourier trans- 
forms of the weights $\left\{w_{n}\right\}$ or $\left\{v_{n}\right\}$ at the spatial frequency $\omega= \pm k d \cos \psi= \pm k L \cos \psi /(N-1)$. Hence, $\omega \rightarrow 0$ as $N \rightarrow \infty$, i.e., the beamforming operation itself provides the required spatial low-pass filtering.

Ignoring mutual coupling between antennas for the moment, one can show formally that (for transmit arrays)

$$
\hat{B}^{\mathrm{T}}(\psi)-B^{\mathrm{T}}(\psi)=O\left(\frac{1}{\sqrt{N}}\right),
$$

where

$$
\hat{B}^{\mathrm{T}}(\psi)=\left|\sum_{n=1}^{N} v_{n} e^{-j n k d \cos \psi}\right| .
$$

The corresponding receive array expressions $\hat{B}^{\mathrm{R}}(\psi)-B^{\mathrm{R}}(\psi)$ are obtained by replacing $j$ with $-j$.

The derivation of (2) has been sketched in [1].

\section{ANTENNA MUTUAL COUPLING AND IMPEDANCE MATCHING}

As more antennas are packed into a fixed aperture, the decreased inter-element separation leads to greater mutual coupling effects. To incorporate antenna mutual coupling into the analysis of the $\Delta \Sigma$ oversampling scheme, we model an $N$-antenna array with an $N$-port characterized by voltages $\mathbf{v} \equiv\left[v_{1} \ldots v_{N}\right]^{T}$ and currents $\mathbf{i} \equiv\left[i_{1} \ldots i_{N}\right]^{T}$. Both are related with an impedance matrix $\mathbf{v}=\mathbf{Z}_{\mathbf{a}} \cdot \mathbf{i}$. Consider perfect conducting, vertically aligned, cylindrical dipole antennas. From the induced EMF method, we can obtain the antenna self-impedances (the diagonal entries of $\mathbf{Z}_{\mathbf{a}}$ ) [12] and mutual impedances of two elements with separation $x$ (the off-diagonal entries of $\mathbf{Z}_{\mathbf{a}}$ ) [13]:

$$
\begin{aligned}
Z_{\text {self }} \approx & \frac{j \eta}{2 \pi} \frac{1}{\sin ^{2}\left(k l_{a} / 2\right)} \int_{0}^{l_{a} / 2} d z \sin \left[k\left(l_{a} / 2-z\right)\right] \times \\
& \left(\frac{e^{-j k \sqrt{\left(z-l_{a} / 2\right)^{2}+r_{a}^{2}}}}{\sqrt{\left(z-l_{a} / 2\right)^{2}+r_{a}^{2}}}+\frac{e^{-j k \sqrt{\left(z+l_{a} / 2\right)^{2}+r_{a}^{2}}}}{\sqrt{\left(z+l_{a} / 2\right)^{2}+r_{a}^{2}}}\right. \\
& \left.-2 \cos \left(k l_{a} / 2\right) \frac{e^{-j k \sqrt{z^{2}+r_{a}^{2}}}}{\sqrt{z^{2}+r_{a}^{2}}}\right)
\end{aligned}
$$

$$
\begin{aligned}
Z_{\text {mutual }}(x) \approx \frac{j \eta}{2 \pi} & \frac{1}{\sin ^{2}\left(k l_{a} / 2\right)} \int_{0}^{l_{a} / 2} d z \sin \left[k\left(l_{a} / 2-z\right)\right] \times \\
& \left(\frac{e^{-j k \sqrt{\left(z-l_{a} / 2\right)^{2}+x^{2}}}}{\sqrt{\left(z-l_{a} / 2\right)^{2}+x^{2}}}+\frac{e^{-j k \sqrt{\left(z+l_{a} / 2\right)^{2}+x^{2}}}}{\sqrt{\left(z+l_{a} / 2\right)^{2}+x^{2}}}\right. \\
& \left.-2 \cos \left(k l_{a} / 2\right) \frac{e^{-j k \sqrt{z^{2}+x^{2}}}}{\sqrt{z^{2}+x^{2}}}\right)
\end{aligned}
$$

The antenna impedance matrix is used to compute the array radiation patterns in the following way: Assume free excitation (which is more realistic in RF systems). Then the actual antenna currents, which are employed to determine the array patterns in (1), are not equal to the forward traveling array signals. Instead, they have to be calculated based on the array signals and impedance matrix from the transmission line theory.

Mutual coupling influences not only radiation patterns but also antennas' power transfer efficiency. Individually matching antenna self-impedances does not warrant an adequate matching network for the array. One must take into account the mutual impedances between antennas. Several approaches of multi-element impedance matching have been proposed, such as eigen-value decomposion and multi-port conjugate matching [14-16]. Although these methods are mathematically sound, they are physically impractical for an array with many elements, as they require extensive interconnectivity between antennas.

Another method is based on the "scan-impedance" of an array. In this method, the matching network is dynamically adjusted to match the active element input impedance for each particular array excitation. In theory, this can provide optimal array efficiency, but at very high complexity costs, since the matching network has to be adjusted whenever the antenna excitation is changed. In this work, we examine a less aggressive version of this method by employing fixed individualized matching networks at all elements. The parameters for these networks are chosen such that the array is perfectly matched for the case of uniform excitations to each array element. The motivation behind this approach is as follows. As the array becomes more densely packed, we expect more severe coupling issues. However, the excitation from one element to the next will also become increasingly uniform. Therefore, designing a matching network based on uniform exitation causes each element to be well matched to its nearest neighbors (the primary source of mutual coupling effects). Further, the $\Delta \Sigma$ outputs will also be increasingly uniform with increasing $N$, implying this seemingly simple matching network is well suited to these complicated conditions. Figs. 3 and 4 illustrate the fixed scan-impedance matching network at the transmitter and receiver, respectively.

\section{SIMULATION RESULTS}

In this section, we present some simulation results. The parameters are set as follows: The wavelength is $\lambda=3 \mathrm{~cm}$ (frequency $10 \mathrm{GHz}$ ). The uniform 1-D array has overall length $L=5 \lambda=15 \mathrm{~cm}$. Each cylindrical antenna has length $l_{a}=$ $1 \mathrm{~cm}$ and radius $r_{a}=1 \mathrm{~mm}$. The signal-feeding transmission lines have characteristic impedance $R_{0}=50 \mathrm{ohm}$, length $l_{t}=0.8 \lambda=2.4 \mathrm{~cm}$, and attenuation $\alpha_{t}=5.0 / \mathrm{m}$. The angular range for beamforming is $\psi \in[0, \pi]$. The matching network is based on the scan impedances for uniform excitations at the transmitter and the receiver. To prevent regular quantization errors that may cause systematic bias on the system, we employ the dithering technique by introducing ran- 


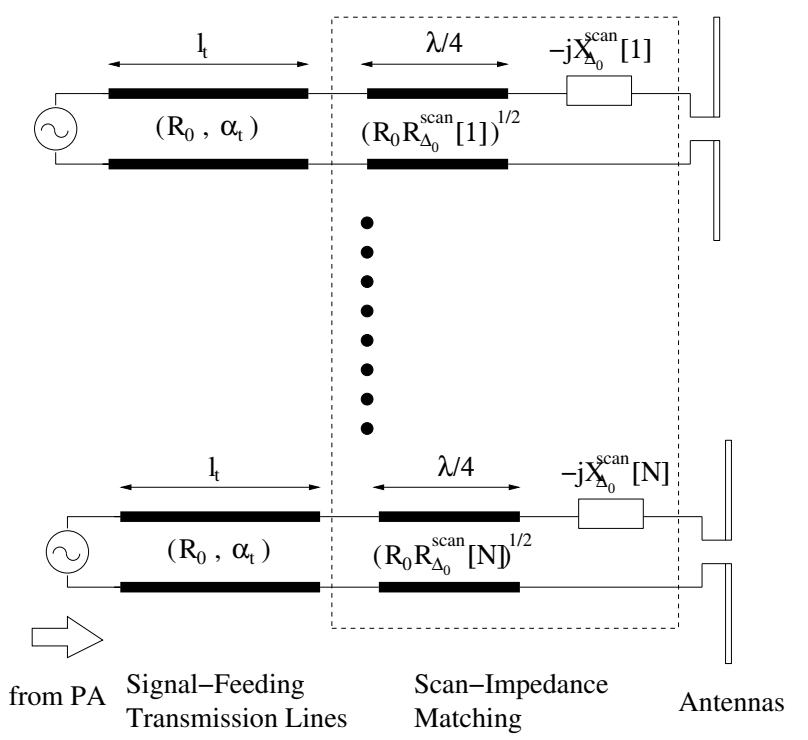

Fig. 3. The architecture of the fixed scan-impedancematching network at the transmitter; $Z_{\Delta_{0}}^{\text {scan }}[n] \equiv R_{\Delta_{0}}^{\text {scan }}[n]+$ $j X_{\Delta_{0}}^{\text {scan }}[n]=\sum_{m=1}^{N} Z_{a}[n, m]$ is the fixed scan impedance of the $n$-th element.

dom phase noise before quantization and the opposite of the same amount of phase shift after quantization. This procedure effectively relaxes our dependence on the particular four quantization levels in the 4-PSQ and hence reduces the chance of regular quantization errors.

\section{Power Transfer Efficiency}

We define the power transfer efficiency for the impedancematching network $\eta_{P} \equiv P_{\text {out }} / P_{\text {in }}$ as the ratio of the output power of the signal-feeding transmssion lines to the input power to the signal-feeding transmission lines. At the transmitter, four matching schemes are compared: the scanimpedance matching, the individualized matching based only on the antennas' self-impedances, no matching at all, and a nearest-neighbor coupled matching. Fig. 5 shows the average power transfer efficiency of the four matching scheme over uniformly distributed beam steering angles at the transmitter. At the receiver, the first three of the four matching schemes are considered. Fig. 6 shows the average power transfer efficiency of the matching scheme at the receiver when the input signal is a plane wave with a random incident angle uniformly distributed within $[0, \pi]$.

In Fig. 5, we can see that the scan-impedance matching outperforms the other matching schemes at most of the $N$ values. The power transfer efficiency under the scan-impedance matching only decreases slightly with $N$, contrasting the other more rapid falls. This implies that the scan-impedance matching can maintain the same power transfer efficiency even when the array becomes much denser. The results in

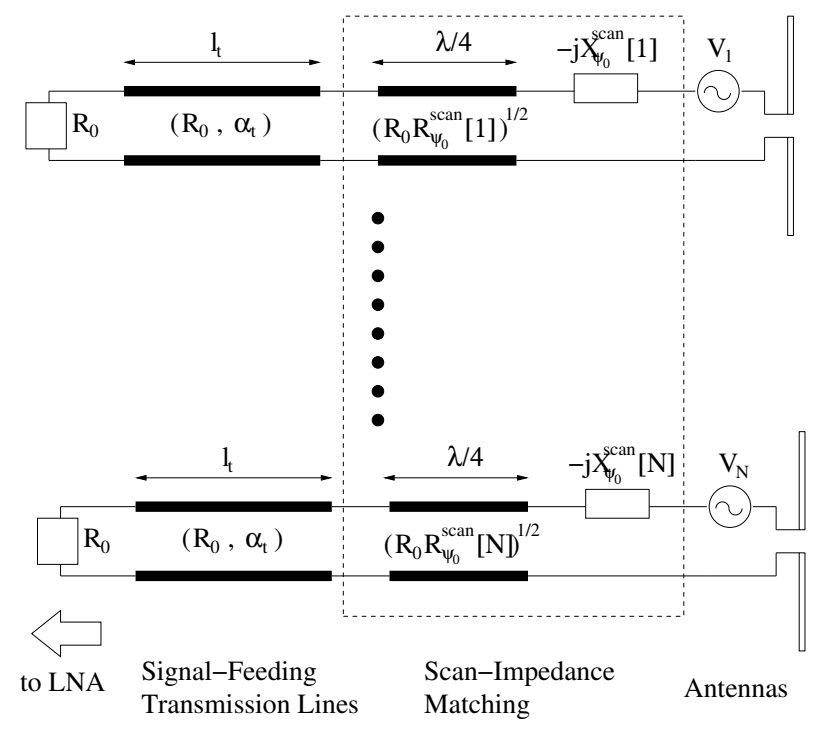

Fig. 4. The architecture of the fixed scan-impedancematching network at the receiver.

Fig. 6 also show that the power transfer efficiency of the scan-impedance matching remains steady when $N$ is large enough, at which it outperforms the other matching schemes.

These results indicate that the scan-impedance matching at the transmitter and the receiver outperforms some common impedance matching networks in power transfer efficiency. Its efficiency does not degrade as $N$ becomes large for some important array inputs we encounter in practice. Thus the scan-impedance matching is a "good-enough" matching scheme for the dense array.

\section{Array Pattern Error}

The antenna pattern error $\|\hat{B}-B\|$ is defined as the difference between the actual array pattern and the ideal array pattern averaged over the looking angle and separable patterns. We compute the array-pattern error when the antenna mutual coupling and the matching network are incorporated. In Fig. 7, we present the numerical results for the transmit phased array under three conditions: scan-impedance matching, no impedance matching, and no antenna mutual coupling. The curve without mutual coupling demonstrates the sheer effect of $\Delta \Sigma$ quantization error, which diminishes toward 0 with $N$ as asserted in Sec. 3 .

Fig. 7 shows that antenna mutual coupling does incur some penalty in convergence rate of the beam pattern quantization error. (The individualized scan-impedance matching does not affect radiation patterns notably.) While a detailed analysis is ongoing, we have found that the mutual coupling effect on pattern errors can be reduced to a multiplicative factor at large $N$, owing to the antenna impedance matrix's Toeplitz structure, i.e., $B^{\text {coupled }}(\psi) \approx C(\psi) B^{\text {uncoupled }}(\psi)$. 


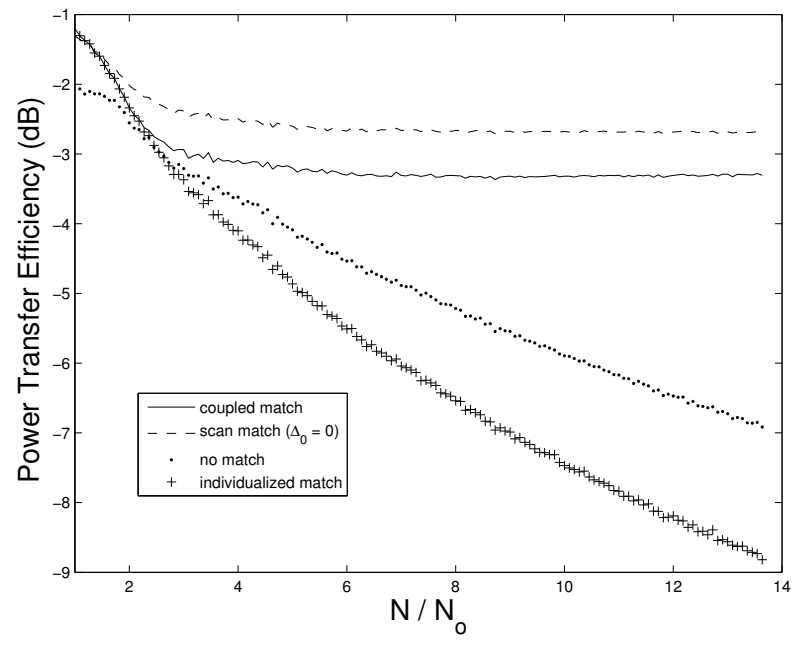

Fig. 5. The power transfer efficiency for the matching networks at the transmitter; phased array; varying $N\left(\Delta_{0}\right.$ denotes the phase angle based on which the fixed scan impedance matching is set; it represents uniform excitation.)

Our calculations indicate that $C(\psi)$ slowly converges to a fairly flat pattern as $N$ becomes large. In any case, the benefits of oversampling are apparent in this analysis.

Compare with the earlier results in [1], the curves in Fig. 7 are considerably smoother. This shows that the dithering technique is effective in reducing the fluctuations of the array pattern errors with respect to the number of antennas.

We can reach the same conclusion for a receive array. Fig. 8 shows the receive array pattern errors with respect to $N$. We compare the case in which the scan-impedance matching and antenna mutual coupling are taken into account with the default case in which none of the two effects are present. Again, the figure shows the benefit of oversampling, in spite of mutual coupling and scan-impedance matching.

\section{CONCLUSIONS}

A dense, spatially oversampling antenna array system can reduce the requirements on the quality of RF devices, including power amplifiers and phase shifters. In such a system, we can reproduce given array radiation patterns with much coarser antenna signals when the number of antennas is large, In this paper, we have elaborated this dense array system developed in [1] by incorporating mutual coupling between antennas and impedance matching. We develop a fixed impedance matching scheme for the dense array based on the array's average scan impedances. This matching scheme is easy to implement, and the preliminary analysis shows that it has "good enough" power transfer performance. Moreover, the antenna array's impedance matrix is computed from electromagnetic theory, and is used to recalculate the array radiation patterns. It can be shown that although mutual coupling indeed incurs

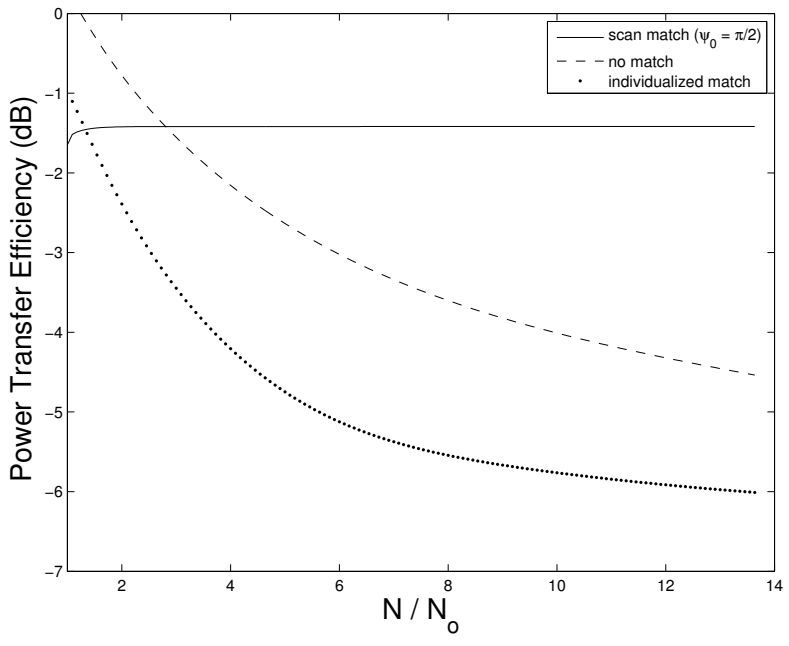

Fig. 6. The power transfer efficiency for the matching networks at the receiver. Plane-wave incidence. Varying $N .\left(\Psi_{0}\right.$ denotes the wave incident angle based on which the fixed scan impedance matching is set; it represents uniform excitation.)

extra array pattern errors, the asymptotic advantage of the dense array in beamforming applications still holds.

\section{ACKNOWLEDEMENT}

This work was supported in part by DARPA under Contract No. HR0011-06-1-0004, and by C2S2 in the FCRP of SRC.

\section{REFERENCES}

[1] C.-P. Yeang, G. W. Wornell, L. Zheng, "Oversampling transmit and receive antenna arrays," Proc. ICASSP, 2010, pp. 2522-2525.

[2] J.-S. Fu and A. Mortazawi, "Improving power amplifier efficiency and linearity using a dynamically controlled tunable matching network," IEEE Trans. Microwave Theory, Techniques, vol. 56, , pp. 3239-3244, Dec. 2008.

[3] S. H. Ji, S. K. Eun, C. S. Cho, J. W. Lee, and J. Kim, "Linearity improved Doherty power amplifier using composite right/left-handed transmission lines," IEEE Microwave, Wireless Components Lett., vol. 18, pp. 533-535, Aug. 2008.

[4] A. van Bezooijen, R. Mahmoudi, and A. H. M. van Roermund, "Adaptive methods to preserve power amplifier linearity under antenna mismatch conditions," IEEE Trans. Circuits, Syst. I, vol. 52, pp. 2101-2108, Oct. 2005. 


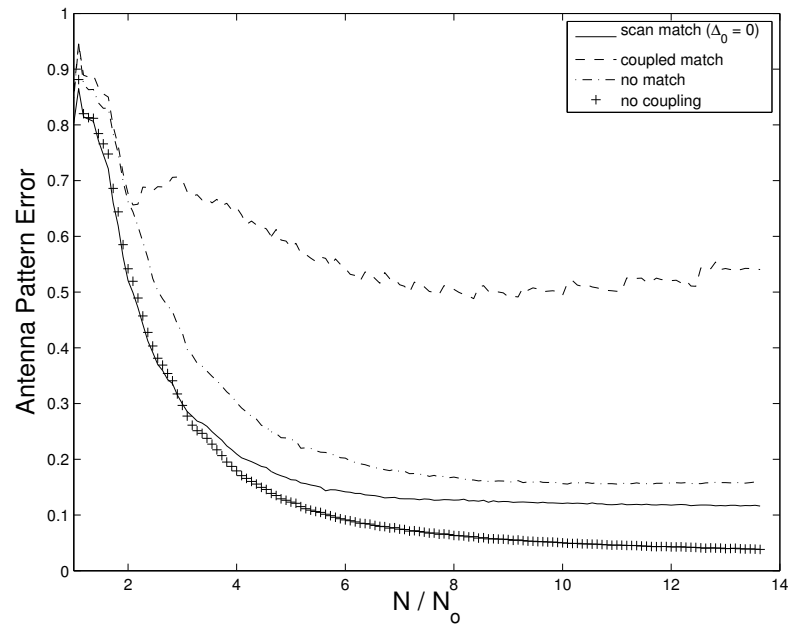

Fig. 7. The array-pattern error at the transmitter. Phased array. With and without mutual coupling and impedance matching.

[5] D. Parker and D. C. Zimmermann, "Phased arrays-Part II: Implementations, applications, and future trends," IEEE Trans. Microwave Theory, Techniques, vol. 50, pp. 688-698, Mar. 2002.

[6] J. Roderick, H. Krishnaswamy, K. Newton, and H. Hashemi, "Silicon-based ultra-wideband beamforming," IEEE J. Solid-State Circuits, vol. 41, pp. 1726-1739, Aug. 2006.

[7] H. Hashemi, X. Guan, A. Komijani, and A. Hajimiri, "A 24-GHz SiGe phased-arry receiver-LO phase-shifting approach," IEEE Trans. Microwave Theory, Techniques, vol. 53, pp. 614-626, Feb. 2005.

[8] S. R. Norsworthy, R. Schreier, and G. C. Temes, DeltaSigma Data Converters: Theory, Design, and Simulation, New York: IEEE Press, 1997.

[9] R. Floyd and L. Steinberg, "An adaptive algorithm for spatial grayscale," Proc. Soc. Image Display, vol. 17, pp. 75-77, 1976.

[10] T. D. Kite, B. L. Evans, A. C. Bovik, and T. L. Sculley, "Digital halftoning as 2-D delta-sigma modulation," Proc. ICIP, 1997, pp. 799-802.

[11] D. P. Scholnik and J. O. Coleman, "Space-time vector delta-sigma modulation," Proc. IEEE Radar Conf., 2004, pp. III-719-722.

[12] R. C. Hansen, Phase Array Antennas, New York: Wiley, 1998.

[13] R. W. P. King, G. J. Fikioris, and R. B. Mack, Cylindrical Antennas and Arrays, Cambridge: Cambridge University Press, 2002.

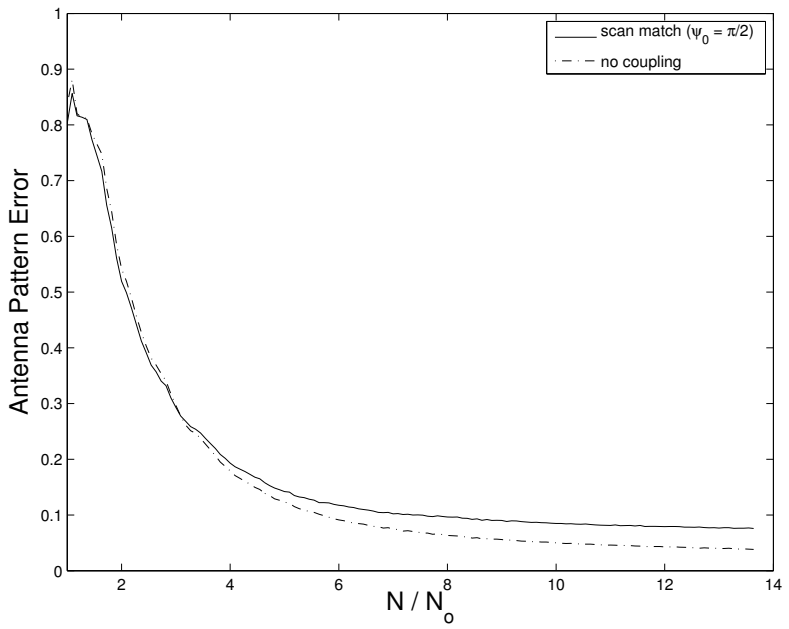

Fig. 8. The array-pattern error at the receiver. Phased array. With and without mutual coupling and impedance matching.

[14] Walter K. Kahn, "Impedance-match and elementpattern constraints for finite arrays," IEEE Trans. Antennas and Propagation, vol. 25, no. 6, pp. 747-755, 1977.

[15] J. Wallace and M. A. Jensen, "Termination-dependent diversity performance of coupled antennas: network theory analysis," IEEE Trans. Antennas and Propagation, vol. 52, no. 1, pp. 98-105, 2004.

[16] J. Wallace and M. A. Jensen, "Mutual coupling in MIMO wireless systems: a rigorous network theory analysis," IEEE Trans. Wireless Communications, vol. 3, no. 4, pp. 1317-1325, 2004. 